\title{
IMPORTANCIA DEL VÍNCULO EXTERNO REMUNERADO EN LA GESTIÓN DE LAS UNIVERSIDADES PÚBLICAS
}

\author{
Isabel Cristina Arroyo Venegas \\ Directora de la Escuela de Administración de Negocios \\ de la Universidad de Costa Rica \\ San José, Costa Rica \\ Liana Penabad Camacho \\ Docente de la Universidad Hispanoamericana \\ San José, Costa Rica
}

Recibido 28-III-2008 • Aceptado 13-V-2008 • Corregido 16-VI-2008

Resumen: En el contexto del siglo XXI, en lo que se refiere al quehacer de las universidades -tanto públicas como privadas- se enfatiza el vínculo Universidad-Sociedad, a través de nuevos enfoques de gestión universitaria que permiten atender las necesidades específicas que plantean los distintos sectores de la sociedad.

En términos generales, las actividades de vínculo externo -remunerado y no remunerado-constituyen, además de una responsabilidad de todas las universidades y en especial las de naturaleza pública, una estrategia efectiva para fortalecer su financiamiento $y$ consecuentemente la gestión universitaria.

El impacto financiero y social del Vínculo Externo Remunerado de las universidades públicas queda evidenciado en el análisis a nivel de ingresos, aportes $e$ inversiones que genera esta actividad dentro del marco de operación, que desde los distintos ámbitos, desarrollan estas instituciones de educación superior.

Palabras clave: Vinculación externa, impacto, Universidad Pública.

\section{Introducción}

Las actividades de vínculo externo -remunerado y no remunerado- constituyen, una responsabilidad de todas las universidades y en especial de las de naturaleza pública, y una estrategia efectiva para fortalecer su financiamiento así como la gestión universitaria a favor de la innovación y mejoras en el proceso educativo que le corresponde atender a estas instituciones en la formación integral de las personas.

En forma específica, el Vínculo Externo Remunerado, en adelante (VER), ha adquirido relevancia en las últimas décadas, a nivel internacional y nacional, por su relación con numerosos sectores a los que les solventa necesidades de primer orden y porque genera una fuente de financiamiento que respalda la ejecución y desarrollo de otras actividades dentro del ámbito universitario y del mismo vínculo externo. 


\begin{abstract}
In the context of the 21st century, and through all the activities of the universities -both state financed or private-there is a primary emphasis in the relationship between University and Society. This is demonstrated through the application of new university management approaches that enable these institutions to attend specific requirements and needs stated by different society groups.

In general terms, the external link activities -remunerated or not-constitute, besides a responsibility for all these universities, and specially for those state financed, an effective strategy to strengthen its financing and consequently the institution's management.

The external remunerated link activities financing and social impact of the state financed universities can be shown through the analysis of revenues, contributions and investment generated in the context of the different activities displayed by these higher education institutions.
\end{abstract}

Key words: External linkage, impact, State Financed University.
Con este artículo se pretende evidenciar el papel que juega el VER dentro de la gestión de una universidad pública y el potencial que este representa como uno de los principales mecanismos de apoyo para optimizar el proceso enseñanza-aprendizaje en la formación de profesionales de las distintas disciplinas del saber.

De ahí nace el interés de incursionar en el análisis de dicha temática, que se desarrolla con respaldo en cuatro apartes en los que se contemplan: la conceptualización e importancia del VER, una contextualización del VER - a nivel internacional y nacional-, el VER como estrategia para fortalecer la relación Universidad-Sociedad, así como el impacto, que a nivel general, produce el VER sobre las universidades públicas.

Es importante mencionar que este artículo antecede a un siguiente artículo, en el que se analiza la temática de la vinculación remunerada con el sector externo para el caso particular de la Universidad de Costa Rica y en el que se incluye una propuesta detallada para mejorar y fortalecer el impacto de esta actividad sobre la gestión estratégica institucional.

\section{Concepto e importancia del VER}

Desde el momento en que una sociedad le abre la posibilidad a una institución de educación superior -en adelante, (IES) - de que lleve a cabo una gestión, esta adquiere el compromiso y la responsabilidad de vincularse con los distintos grupos o sectores de su entorno social en búsqueda de soluciones a problemas y necesidades que conduzcan al bien común. En ese sentido, la claridad en la conceptualización de la actividad se vuelve importante para garantizar el éxito en la consecución de este compromiso.

Si bien no existe una definición explícita del concepto de Vinculo Externo -remunerado o no remunerado-se entiende por éste toda actividad en la que participan 
dos tipos de actores: el prestatario u oferente de la actividad de vinculación, a quien le corresponde su planeación, dirección y ejecución, y el usuario o beneficiario quien recibe un bien o servicio para suplir una necesidad o alcanzar un objetivo o meta específica. Por otro lado; se trata, también, de una relación cuyo propósito es generar beneficios recíprocos a las partes involucradas, en condiciones que le permitan, a cada una, realizar el mejor esfuerzo y medir de forma equilibrada y justa los resultados del vínculo establecido.

De manera específica, el VER cuenta con una retribución en recursos financieros que es aportada por los beneficiarios o por otros actores externos que desean, por la vía de la donación o aporte, contribuir con los objetivos o metas que enmarcan estas actividades. El Vínculo Externo No Remunerado, en adelante (VENR), no cuenta con una retribución directa de los beneficiarios o agentes externos y sus actividades son financiadas con recursos propios del prestatario $u$ oferente, que para efectos de este documento debe entenderse como las universidades.

En términos generales, se dan diversas categorizaciones de las actividades de vínculo externo en las universidades; ellas están en función de la naturaleza particular de cada una y toman en consideración las áreas sustantivas de la academia. Esta clasificación constituye una base tanto para el proceso de planificación como para la medición de impacto y emisión de informes de gestión.

Por otro lado, entre los diferentes enfoques y criterios, planteados por académicos o instancias universitarias, se encuentran algunas concepciones que orientan la definición de Vinculación Externa y Vinculación Externa Remunerada; por ejemplo, la Oficina de Planificación de la Educación Superior, en adelante (OPES), en el documento: $L a$ vinculación de las universidades estatales costarricenses con el sector producti$v o$, cita que la vinculación con la sociedad se refiere a: "aquellas tareas donde la institución educativa es soporte para la solución de problemas de la sociedad" (CONARE-OPES, 2004, p. 3).

Además, establece una diferencia entre extensión y vinculación, ambas como funciones que propician un acercamiento entre las IES y la sociedad y reconoce la necesidad de redefinir estos conceptos, tal que se entienda:

... la extensión como la transmisión del quehacer universitario, más allá de su acción intramuros. En este sentido es la acción vinculadora de la universidad con su entorno, si esta responde a las necesidades de la población y existen beneficios mutuos entre las partes ... la vinculación la entendemos como el conjunto de relaciones entre las instituciones de educación superior con los sectores público, privado y social, a través de instrumentos legales apropiados, para conocer y ofrecer los frutos del quehacer universitario; el vehículo para poner al alcance de la sociedad la prestación de servicios universitarios ... la vinculación tiene implícita una función extensora: acercar los frutos del quehacer universitario a todos los actores de la sociedad (CONARE-OPES, 2004, p. 4) ${ }^{1}$.

Además, una Comisión Especial de académicos creada por el Consejo Universitario de la Universidad de Costa Rica establece sobre la definición de vinculación externa:

La venta de bienes y la prestación remunerada de servicios constituye una modalidad de vinculación de la Universidad de Costa Rica con el sector externo, de la que son susceptibles programas o proyectos de las dimensiones sustanciales del quehacer académico (docencia, investigación y acción social), caracterizada por incluir el aporte económico del usuario en su financiamiento. Es una actividad derivada del quehacer académico universitario, cuyo propósito es contribuir al desarrollo nacional y enriquecer, a la vez, el desarrollo de la Universidad (Consejo Universitario de la Universidad de Costa Rica, Comisión Especial, 2004, p. 3).

Según los Lineamientos para la vinculación remunerada de la Universidad de Costa Rica con el sector externo, el concepto del VER debe plantearse desde diferentes perspectivas tal y como se resume en el siguiente diagrama: 


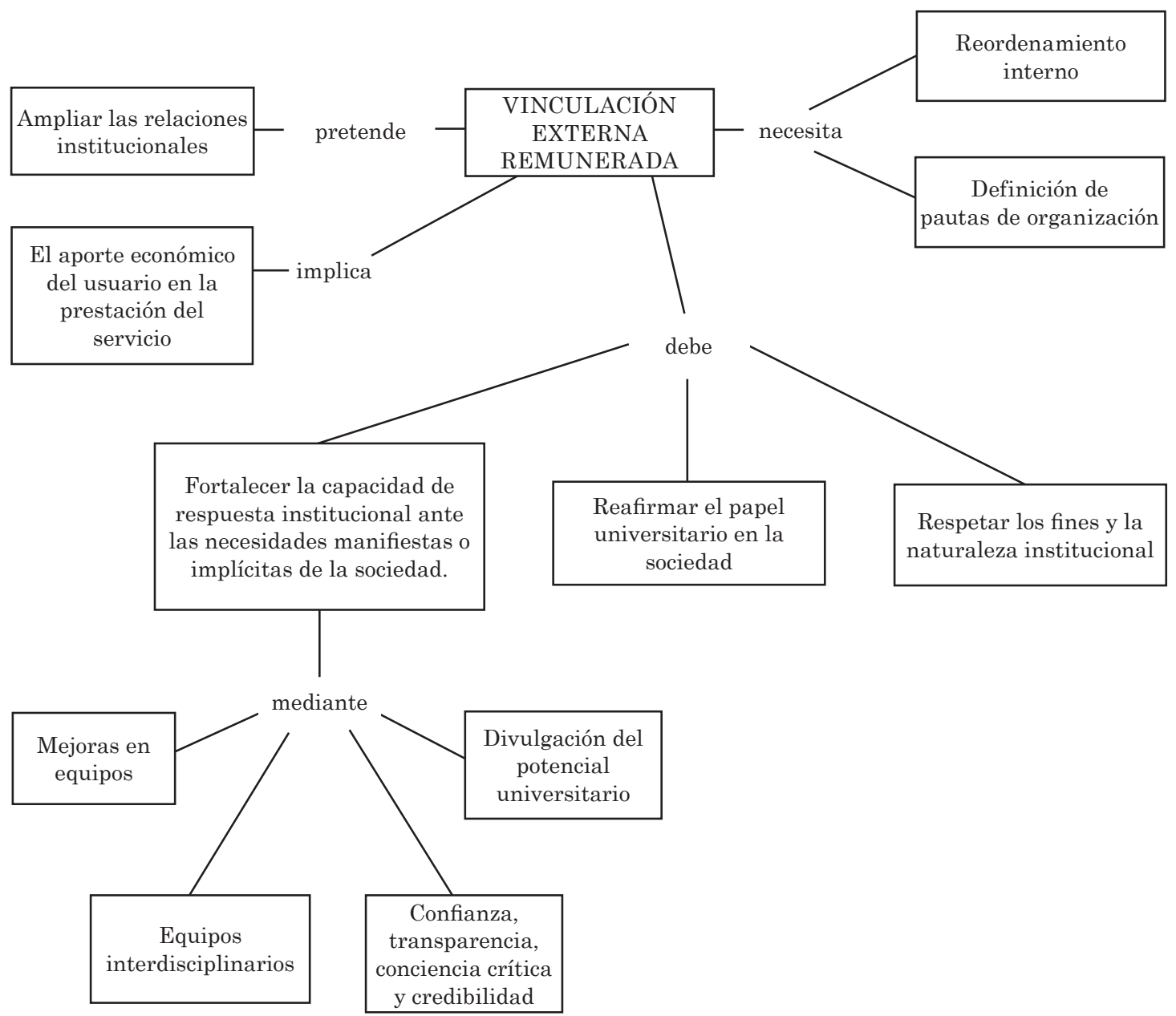

Fuente: Lineamientos para la vinculación remunerada de la Universidad de Costa Rica con el sector externo.

La visualización mostrada en el diagrama anterior plantea claramente que el VER de la Universidad de Costa Rica debe buscar una mayor apertura de la Institución con los distintos actores sociales; alinearse con su marco estratégico y fortalecer el impacto institucional sobre los distintos sectores de la comunidad para lo que requiere un reordenamiento a nivel interno.

Se puede deducir de lo planteado en los párrafos anteriores que la misión declarada para todas las universidades del nuevo siglo evidencia la importancia y la necesidad de una gestión vinculada con los diferentes sectores de la sociedad, donde revistan especial importancia los objetivos relacionados con las actividades de vínculo externo, pues son las que abren posibilidades reales para todos los ciudadanos que no tienen la opción de ingresar al sistema educativo superior formal.

Al tomar en cuenta las diversas concepciones adoptadas para el VER, se puede concluir que esta actividad se convierte en una vía que le permite a las universidades solventar-en alguna medidalas limitaciones presupuestarias que se les 
presentan, como instituciones públicas con un presupuesto proveniente del Estado y les facilita ejecutar acciones congruentes con la misión y objetivos contemplados en sus marcos estratégicos. De la misma forma, el VER se constituye en una buena opción para fortalecer la relación universidad-sociedad porque permite la generación de soluciones a diversos problemas y necesidades que enfrentan los distintos sectores que la conforman.

\section{Contextualización del VER}

La vinculación externa puede ser considerada como una estrategia para permitir igualdad en el acceso a la producción del conocimiento científico y tecnológico y para que: “...las instituciones de educación superior asuman mayores responsabilidades para con la sociedad y rindan cuentas sobre la utilización de los recursos públicos y privados, nacionales o internacionales" (UNESCO, 1998, p. 75).

\section{Contexto internacional}

De acuerdo con la UNESCO, en su Declaración de París 1998, el VER potencia el desarrollo de las capacidades de investigación, técnicas y tecnológicas presentes en el entorno universitario y fomenta la participación de distintos actores de los sectores público y privado de la sociedad. En ese sentido, afirma que las IES: "deberían obtener el apoyo material y financiero necesario de fuentes públicas y privadas" (UNESCO, 1998, p. 78).

Se estima que ha habido consenso entre todas las universidades del mundo, por medio de la UNESCO, en que se debe reforzar la cooperación con el ámbito laboral y demás grupos de la comunidad, así como el análisis y las previsiones de las necesidades que estos presenten. Específicamente en el Artículo 7, inciso a) la Declaración señala:
...En un contexto económico caracterizado por los cambios y la aparición de nuevos modelos de producción basados en el saber y sus aplicaciones, así como en el tratamiento de la información, deberían reforzarse y renovarse los vínculos entre la enseñanza superior, el mundo del trabajo y otros sectores de la sociedad (UNESCO, 1998, p. 79).

Además, se ha evidenciado el acuerdo que existe con respecto a la necesidad de diversificar las fuentes de financiamiento en las universidades: “...a fin de garantizar el desarrollo de este tipo de enseñanza, de aumentar su eficacia y de mantener su calidad y pertinencia..." (UNESCO, 1998, p. 83).

Estudios realizados sobre la estructura de financiamiento de universidades de Estados Unidos, Europa y China ${ }^{2}$ demuestran una clara tendencia a la diversificación de sus fuentes de ingresos. Independientemente de si se trata de universidades públicas o privadas, la participación del sector privado por medio de actividades de vinculación remunerada ha tomado especial importancia y experimentado un crecimiento en las dos últimas décadas. En ese sentido, se considera que el aumento del VER no solo amplía la base de los ingresos sino la capacidad de retroalimentación para el fortalecimiento del proceso educativo y en general de la relación Universidad-Sociedad por medio de la amplia oferta de bienes y servicios que se generan para la atención de necesidades y la resolución de problemas.

\section{Contexto nacional}

En el ámbito de la educación superior costarricense, las tendencias y papel de las actividades de vinculación externa, con su respectivo impacto en el presupuesto de las universidades, es muy similar a lo analizado en el contexto internacional.

En el contexto nacional desarrollan actividades de vinculación externa -remunerada y no remunerada- las cuatro universidades públicas y varias 
universidades privadas, estas últimas dedican la mayor parte de sus esfuerzos al campo de la capacitación y actualización. Las universidades públicas tienen una oferta diversificada y sus esfuerzos están orientados tanto al área de investigación como al campo social, mediante servicios como asesorías, asistencias, capacitación y actualización profesional.

CONARE, en el Plan para la Educación Superior Estatal 2006-2010 (PLANES), contempla un primer eje estratégico de gestión, denominado Pertinencia e Impacto, dentro del cual establece varios objetivos estratégicos, relacionados con la vinculación de alcance nacional e internacional. En ese marco, como su primer objetivo estratégico, señala para el campo nacional:

Fomentar mecanismos de vinculación del quehacer sustantivo de la educación superior universitaria estatal con diversos órganos de los sectores público y privado y con la economía nacional (CONARE-OPES, 2005, p. 35).

Además, los Objetivos Estratégicos 12 y 13 indican, en lo relacionado con el campo internacional:

12. Promover y fortalecer lazos de cooperación con entes externos, para impulsar la difusión, innovación y docencia, la investigación, la extensión y la acción social.

13. Fortalecer vínculos en el ámbito internacional para incentivar el intercambio de experiencias, proyectos y programas académicos conjuntos, que aseguren una interrelación con equidad (CONARE-OPES, 2005, p. 38).

Además, en informes de gestión de las cuatro universidades públicas se evidencia el crecimiento y la importancia que ha alcanzado el VER. Si se parte de los resultados que muestran los estados financieros - principalmente de las universidades estatales- es palpable la diversificación y el impacto que genera el VER en las estructuras de financiamiento y la gestión global de cada institución.

Los Estatutos Orgánicos de las cuatro universidades estatales manifiestan el com- promiso y la responsabilidad que tienen con la sociedad, cuyo cumplimiento se apoya en buena medida en las actividades de vinculación remunerada. Así mismo, las cuatro universidades disponen de fundaciones, como mecanismo facilitador en la administración de los recursos que genera la vinculación con el sector externo, tal como lo señala el documento La vinculación de las universidades estatales costarricenses con el sector productivo (CONARE-OPES, 2004).

\section{El VER como estrategia para fortalecer la relación Universidad-Sociedad}

Al considerar la oferta de bienes y servicios que genera el VER, a nivel mundial, para diversas actividades que fomentan el desarrollo socioeconómico de un país y en múltiples campos del conocimiento y aplicación, se puede aseverar que este ámbito de la gestión universitaria es fundamental en la relación Universidad-Sociedad.

En ese sentido, y como resultado del estudio que respalda este artículo, se identificaron las siguientes fortalezas del VER:

- $\quad$ Fomenta el trabajo interdisciplinario e incrementa la capacidad de respuesta de las universidades.

- Permite una mayor articulación entre las dependencias universitarias y los diferentes actores sociales involucrados.

- Propicia la unión de esfuerzos y recursos para el logro de objetivos comunes.

- Amplía y diversifica opciones para la solución de problemas sociales.

- Genera un efecto expansivo de los alcances y beneficios derivados del quehacer universitario.

Por consiguiente, el VER siempre podrá considerarse como una buena estrategia universitaria para mantener una 
sólida y renovada relación con la sociedad y para coadyuvar en el cumplimiento de la misión institucional.

\section{Impacto del VER sobre la gestión de las universidades públicas}

Tal como lo respalda la Declaración de la UNESCO (UNESCO, 1998), y los resultados de la gestión de las distintas universidades del mundo, que se comentan en distintos informes y reportes encontrados en Internet, la vinculación remunerada de estas organizaciones no solo constituye una necesidad -para que los países y sus diferentes sectores logren los niveles de competitividad y eficiencia que le demanda la sociedad- sino una opción válida para fortalecer sus funciones sustantivas y su estructura de financiamiento.

En general, las instituciones de educación superior a nivel mundial han avanzado en la visión de su labor fundamental pues asumen un papel relevante y protagónico en el desarrollo de los diferentes sectores sociales, dentro de lo que se ha denominado "la sociedad mundial de conocimiento", donde la globalización exige la transferencia de respuestas más concretas, la creación de alianzas y una mayor accesibilidad a los beneficios del quehacer universitario, especialmente por parte de los grupos más vulnerables o de aquellos que poseen el potencial pero, no tienen la oportunidad de desarrollarse por sus propios medios.

Toda vinculación de una universidad con sectores externos, más allá de lo que representa la formación de profesionales y de la investigación de apoyo a esa formación, además de una acción social primaria -sobretodo en las instituciones de carácter público- requiere la inversión de recursos importantes que difícilmente pueden extraerse de los aportes de cada gobierno.

En resumen, hay una clara tendencia que ha llevado a un cambio de cultura, paradigmas y enfoques de gestión, que evidencian un fortalecimiento de las actividades del Vínculo Externo, que están obligadas a ejecutar todas las IES, para evitar un desequilibrio y debilitamiento en la misión que deben cumplir como gestoras claves del desarrollo socioeconómico de las naciones.

El impacto del VER sobre la gestión de las universidades se puede visualizar desde dos perspectivas o ámbitos: financiero y social.

\section{Impacto financiero}

Desde la perspectiva financiera, se pueden citar algunos ejemplos de la participación del VER en la gestión de algunas universidades o sistemas universitarios del mundo.

En el caso de la Universidad de Cape Town, Sudáfrica, en su reporte anual, de acuerdo con sus estados financieros 2004-2005, aproximadamente un 27\% de sus ingresos provienen de rubros que se pueden relacionar con actividades de VER; ese año el aporte del Estado representó el $35 \%$ y los ingresos por matrícula un $26,7 \%$ (Universidad de Cape Town, 2005).

Un estudio llevado a cabo por Xin Wang de la Universidad de Baylor, USA, sobre el desempeño del sistema universitario chino durante dos décadas, indica que para 1978 solo un $4,1 \%$ de los ingresos universitarios provinieron de vinculación externa, mientras que en 1992 esta generó un 13,6\% de los ingresos; el aporte del gobierno fue un $99,5 \%$ en 1978 y un $81,8 \%$ en 1992 (Wang, 2001).

En el panorama de las universidades estatales en Estados Unidos se citarán dos ejemplos. En el caso de la Universidad Estatal de Arizona, según su Informe de gestión financiera del año 2006, las actividades de vinculación externa representan un $26 \%$ y en la Universidad de Kansas, durante el 2006, este rubro representó el $28 \%$. 
Por su parte la Universidad Nacional Autónoma de México (UNAM), reporta en su presupuesto correspondiente al año 2006, un monto en actividades de Vinculación Externa que representa el $8,8 \%$ del total de sus ingresos, el Subsidio del Gobierno Federal representó un 88,9\% (UNAM, 2007).

En lo que se refiere al contexto costarricense las actividades del VER son desarrolladas principalmente por las cuatro universidades estatales, dado que; el peso de las que desarrollan las universidades privadas no es relevante, ya que solo se concretan algunos cursos de actualización con muy poca prestación de servicios o productos comunes al quehacer académico.

A partir de la información financiera obtenida de las cuatro universidades públicas, se elaboró el Cuadro 1 y su respectivo Gráfico 1, que evidencian el importante rol que juegan todas las actividades de vinculación remunerada y la participación que en éstas tiene cada institución.

Se desprende de esta información que del total de ingresos generados por el VER un $72 \%$ correspondió a la Universidad de Costa Rica, un 13\% al Instituto Tecnológico de Costa Rica, un $13 \%$ a la Universidad Nacional y un $2 \%$ a la Universidad Estatal a Distancia. Por otra parte, en el caso de la Universidad de Costa Rica, los ingresos generados por el VER en el año 2006 representaron un $17 \%$ de sus ingresos totales, monto que permitió, además de importantes inversiones para el fortalecimiento del proceso educativo, el financiamiento de diversas actividades de vinculación no remuneradas en beneficio de varios sectores vulnerables del país.

\section{Impacto social}

Si se analizan los datos relacionados con la diversidad y el número de proyectos de vinculación remunerada que realizan las universidades públicas, sin duda alguna, se evidencia la cantidad de ideas y esfuerzos desplegados desde las distintas áreas de gestión y desde todas las áreas del saber que permiten visualizar, en un primer

Cuadro 1

Vínculo externo remunerado Universidades públicas Periodo 2006 (en colones)

\begin{tabular}{lccccc}
\hline Institución & $\begin{array}{c}\text { Ingresos } \\
\text { Totales }\end{array}$ & $\begin{array}{c}\text { Participación } \\
\%\end{array}$ & $\begin{array}{c}\text { Ingresos } \\
\text { Docencia }\left(^{*}\right)\end{array}$ & $\begin{array}{c}\text { Ingresos } \\
\text { Netos }\end{array}$ & $\begin{array}{c}\text { Participación } \\
\%\end{array}$ \\
\hline UCR & 15.551 .905 .681 & 67 & 957.483 .103 & 14.594 .422 .578 & 72 \\
ITCR & 2.901 .692 .856 & 13 & 301.583 .297 & 2.600 .109 .559 & 13 \\
UNA & 3.902 .243 .811 & 17 & 1.201 .247 .849 & 2.700 .995 .962 & 13 \\
UNED & 793.250 .000 & 3 & 450.520 .000 & 342.730 .000 & 2 \\
\hline Totales & 23.149 .092 .348 & 100 & 2.910 .834 .249 & 20.238 .258 .100 & 100 \\
\hline
\end{tabular}

(*) Estosingresosincluyentodoslosprogramasdepregrado,gradoyposgradoconfinanciamientocomplementario.

Fuente: Informes financieros y de labores proporcionados por las Rectorías, Fundaciones o Dependencias financieras de las cuatro Universidades públicas 
Gráfico 1

VínculoExterno Remunerado Universidades Públicas Periodo 2006

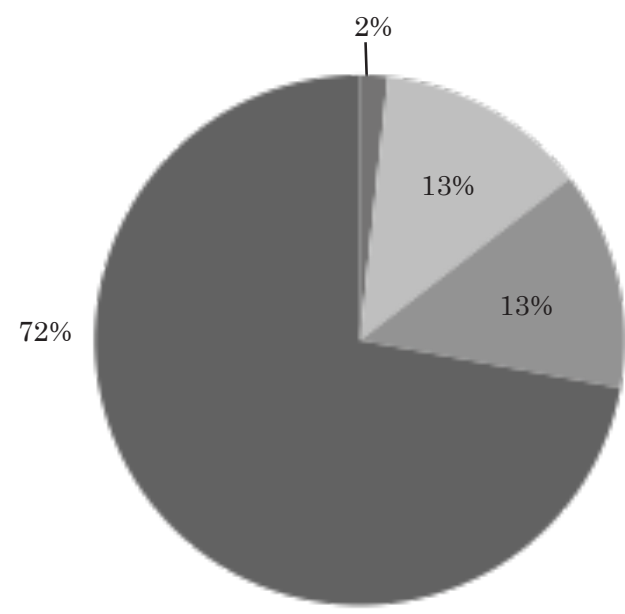

UCR

ITCR

UNA

UNED

acercamiento, la magnitud de un impacto social desde la perspectiva de la generación y transmisión de conocimiento, que produce la vinculación remunerada y que se traduce en beneficio directo para muchos grupos y sectores de la sociedad: privados, públicos, por género, empresariales, de todas las clases sociales, etc. Varios de estos grupos reciben un beneficio producto de un proyecto o actividad en la que se reúnen diversas áreas del conocimiento, que generan un esfuerzo interdisciplinario con el fin de darle solución a importantes problemas sociales.

De acuerdo con lo planteado, el impacto social constituye el fin último del Vínculo Externo Remunerado; sin embargo, es la dimensión que menos está evaluada y la que, en el nivel de sistemas de información y rendición de cuentas, está en un segundo plano, lo que consecuentemente le resta importancia al impacto total del VER sobre la Gestión Estratégica universitaria y sobre el desarrollo de los distintos grupos y sectores de la sociedad.

\section{Reflexión Final}

La vinculación de las IES con los sectores externos de la sociedad, por medio de actividades remuneradas, ha experimentado un crecimiento en el contexto internacional y también a nivel de las universidades estatales costarricenses -sobretodo en las últimas décadas- provocado por la necesidad de diversificar las oportunidades de acceso a los beneficios derivados del quehacer universitario y de obtener el apoyo financiero de fuentes públicas y privadas para responder solidariamente a las demandas de la sociedad.

A partir de las cifras financieras y de la diversidad y número de proyectos que reportan diferentes universidades en distintos periodos, se confirma, el importante rol que juegan todas las actividades de vinculación remunerada desarrolladas por estas instituciones.

Desde distintos puntos de vista el VER constituye un eje importante y necesario para promover la sana competitividad, el desarrollo de las capacidades humanas y mejorar la calidad de vida de las personas. No obstante lo anterior, es claro que todavía se requieren estrategias y acciones de fortalecimiento y mejora que permitan un adecuado seguimiento y medición de impacto de estas actividades, con el fin de potenciar los beneficios que se derivan de ellas.

\section{Notas}

1. La Oficina de Planificación de la Educación Superior (OPES) cita a http://www. ugcarmen.edu.co/Revista\%20Futuro/ vinculacion/20universidad.htm

2. Informes anuales de gestión de diferentes universidades a nivel mundial y financiamiento, gestión y nuevos escenarios para la producción de conocimientos: "Financiamiento, gestión y nuevos escenarios para la producción de conocimientos" (Rosell, L., 2004), "Financiamiento y gestión de la enseñanza superior: informe sobre los progresos de las reformas en el mundo" (Johnstone, B., Arora, A. y Experton, W. (1998), 
para el Banco Mundial y "A policy analysis or the finanacing of higher education in China: two decades reviewed" (Wang, X, 2001).

\section{Referencias bibliográficas}

Consejo Nacional de Rectores [CONARE]. Oficina de Planificación de la Educación Superior [OPES]. (2004). La vinculación de las universidades estatales costarricenses con el sector productivo. San José, Costa Rica: CONARE-OPES, Publicaciones.

Consejo Nacional de Rectores [CONARE], Oficina de Planificación de la Educación Superior [OPES]. (2005). Plan Nacional de la Educación Superior Universitaria Estatal, [PLANES] 2006-2010. San José, Costa Rica: CONARE-OPES.

Consejo Universitario, Comisión Especial. (2004). Vinculación de la Universidad de Costa Rica con el sector externo mediante la venta de bienes $y$ la prestación remunerada de servicios. San José, Costa Rica: Consejo Universitario de la Universidad de Costa Rica.

Johnstone, B., Arora, A. y Experton, W. (1998). Financiamiento y gestión de la enseñanza superior: informe sobre los progresos de las reformas en el mundo. Washington: Banco Mundial.
Organización de las Naciones Unidas para la Educación, la Ciencia y la Cultura [UNESCO]. (1998, 9 de octubre). Declaración Mundial sobre la Educación Superior en el siglo XXI: Visión y acción. Boletín 47, Proyecto Principal de Educación, 73-91.

Rosell, L. (2004). Financiamiento, gestión y nuevos escenarios para la producción de conocimientos. Fundación Acta Odontolólgica Venezolana. Artículo 8. Consultado el 14 de abril de 2007 de http://www.actaodontologica. com/44_2_2006/produccion_ conocimientos.asp

Universidad de Cape Town. (2005). Annual Report [Reporte anual]. Cape Town, Sudáfrica: Universidad de Cape Town.

Universidad Nacional Autónoma de México. (2007). Presupuesto 2006. Extraído el 14 de mayo de 2007 de http://www. planeacion.unam.mx/agenda/2006/ pdf/26_presupuesto06.pdf

Wang, X. (2001). A policy analysis or the financing of higher education in china: two decades reviewed. [Un Análisis de Políticas de Financiación de la Educación Superior en China: dos décadas revisadas]. Journal of Higher Education Policy and Management, 2(23), 18-27. 